\title{
Geometric similarity measures for the intuitionistic fuzzy sets
}

\author{
Eulalia Szmidt and Janusz Kacprzyk \\ Systems Research Institute Polish Academy of Sciences, ul. Newelska 6, 01-447 Warsaw, Poland \\ WIT - Warsaw School of Information Technology, ul. Newelska 6, 01-447 Warsaw, Poland
}

\begin{abstract}
This paper is a continuation of our previous works on geometric similarity measures between Atanassov's intuitionistic fuzzy sets (A-IFSs for short). We consider some traps of the straightforward approach in the case of A-ISs while similarity is understood as a dual concept of a distance. The difficulties are a result of, first, the symmetry of the three terms (the membership, nonmembership and hesitation margin) in an A-IFS element description, and second, of an important role played by those three terms in the definition of the complement of the A-IFS which should be taken into account in the similarity measures.
\end{abstract}

Keywords: Intuitionistic fuzzy sets, distances, similarity measures

\section{Introduction}

It is not possible to overestimate the importance of similarity measures - they are used in many algorithms, but even the best algorithm can not produce satisfactory results when similarity measures used are not reliable.

This paper is continuation of our previous works on distances and similarity measures for A-IFSs (cf. Szmidt and Kacprzyk [15], [18], [17], [19], [20], [21], [24]). We consider similarity at the basic level, i.e., similarity of the elements belonging to an A-IFS, characterized by the membership, non-membership and hesitation margin, and next - similarity of the A-IFSs assuming that their elements are represented geometrically (as points in a coordinate space). Similarity is usually assumed to be a dual notion to a distance. However the adequacy of the geometric approach with its assumptions (symmetry, transitivity, ...) especially from a psychological point of view changes for different practical situations (sometimes a property is useful, sometimes undesirable). For example, we can say (cf. Tversky [30]): "the portrait resembles the person" rather than "the person resembles the portrait". Anyway, the geometric approach is rather popular and has been successfully applied to real problems (e.g., Carroll and Wish [8], Shepard [11]).

In this paper we consider if straightforward geometrical approach to similarity (as a dual notion of a distance) is sufficient for considering similarity between the AIFSs. After pointing out some difficulties we emphasize the necessity of including into the definition of similarity a concept of a so called complement element introduced by Atanassov [3] to be presented in Section 2. We consider some similarity measures with different types of distances.

\section{A brief introduction to the A-IFSs}

One of the possible generalizations of a fuzzy set in $X$ (Zadeh [34]) given by

$$
A^{\prime}=\left\{<x, \mu_{A^{\prime}}(x)>\mid x \in X\right\}
$$

where $\mu_{A^{\prime}}(x) \in[0,1]$ is the membership function of the fuzzy set $A^{\prime}$, is an A-IFS (Atanassov [1], [3], [4]) $A$ is given by

$$
A=\left\{<x, \mu_{A}(x), v_{A}(x)>\mid x \in X\right\}
$$

where: $\mu_{A}: X \rightarrow[0,1]$ and $v_{A}: X \rightarrow[0,1]$ such that

$$
0 \leq \mu_{A}(x)+v_{A}(x) \leq 1
$$

and $\mu_{A}(x), v_{A}(x) \in[0,1]$ denote a degree of membership and a degree of non-membership of $x \in A$, respectively.

Obviously, each fuzzy set may be represented by the following A-IFS

$$
A=\left\{<x, \mu_{A^{\prime}}(x), 1-\mu_{A^{\prime}}(x)>\mid x \in X\right\}
$$

An additional concept for each A-IFS in $X$, that is not only an obvious result of (2) and (3) but which is also relevant for applications, we will call (Atanassov [3])

$$
\pi_{A}(x)=1-\mu_{A}(x)-v_{A}(x)
$$

a hesitation margin of $x \in A$ which expresses a lack of knowledge of whether $x$ belongs to $A$ or not (cf. Atanassov [3]). It is obvious that $0 \leq \pi_{A}(x) \leq 1$, for each $x \in X$.

The hesitation margin turns out to be important while considering the distances (Szmidt and Kacprzyk [14], [15], [19], entropy (Szmidt and Kacprzyk [16], [22]), similarity (Szmidt and Kacprzyk [21]) for the A-IFSs, etc. i.e., the measures that play a crucial role in virtually all information processing tasks.

Hesitation margins turn out to be relevant for applications - in image processing (cf. Bustince et al. [7], [6]) and classification of imbalanced and overlapping classes (cf. Szmidt and Kukier [27], [28], [29]), group decision making, negotiations, voting and other situations (cf. Szmidt and Kacprzyk papers). 


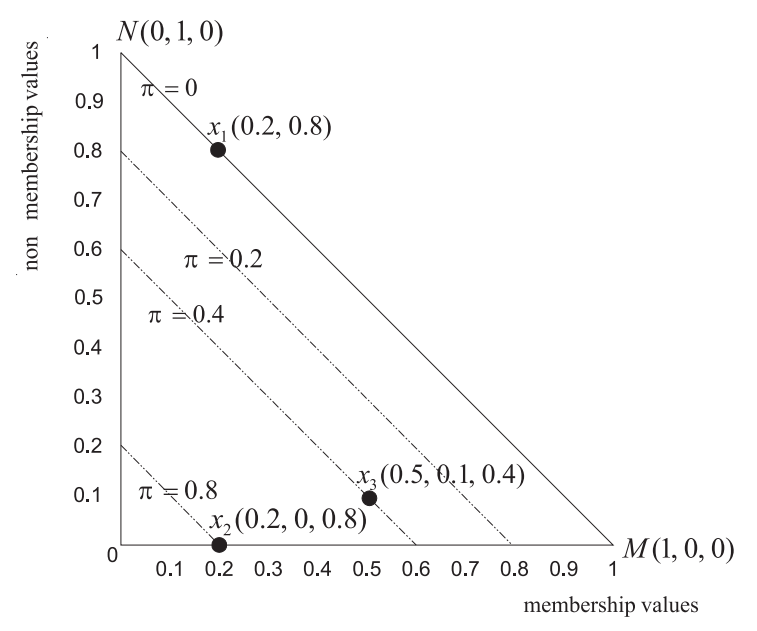

Figure 1: Geometrical representation

In our further considerations on similarity we will use the notion of the complement of an A-IFS, denoted by $A^{C}$, and defined as (cf. [3]):

$$
A^{C}=\left\{<x, v_{A}(x), \mu_{A}(x)>\mid x \in X\right\} .
$$

and $x:\left(v_{A}(x), \mu_{A}(x)\right)$ is called a complement element.

\subsection{A geometrical representation}

One of possible geometrical representations of an A-IFS is given in Figure 1 (cf. Atanassov [3]). It is worth noticing that although we use a two-dimensional figure (which is more convenient to draw in our further considerations), we still adopt our approach (e.g., Szmidt and Kacprzyk [15], [19], [16], [22]), [21]) taking into account all three terms (membership, non-membership and hesitation margin values) describing an A-IFS. Any element belonging to an A-IFS may be represented inside an $M N O$ triangle. In other words, the $M N O$ triangle represents the surface where the coordinates of any element belonging to an A-IFS can be represented. Each point $x$ belonging to the $M N O$ triangle is therefore described by the respective values on the three coordinates: $\left(\mu_{A}(),. v_{A}(),. \pi_{A}().\right)$. Points $M$ and $N$ represent the crisp elements. Point $M(1,0,0)$ represents elements fully belonging to an A-IFS as $\mu=1$, and may be seen as the representation of the ideal positive element. Point $N(0,1,0)$ represents elements fully not belonging to an A-IFS as $v=1$, i.e. can be viewed as the ideal negative element. Point $O(0,0,1)$ represents elements about which we are not able to say if they belong or not belong to an A-IFS (the intuitionistic fuzzy index $\pi=1$ ). Such an interpretation is intuitively appealing and provides means for the representation of many aspects of imperfect information. Segment $M N$ (where $\pi=0$ ) represents elements belonging to the classic fuzzy sets $(\mu+v=1)$. For example, point $x_{1}(0.2,0.8,0)$ (Figure 1), like any element from segment $M N$ represents an element of a fuzzy set. A line parallel to $M N$ describes the elements with the same values of the hesitation margin. In Figure 1 we can see point $x_{3}(0.5,0.1,0.4)$ representing an element with the hesitation margin equal 0.4 , and point $x_{2}(0.2,0,0.8)$ representing an element with the hesitation margin equal 0.8 . The closer a line that is parallel to $M N$ is to $O$, the higher the hesitation margin.

\section{Similarity measures between the A-IFSs}

In the case of fuzzy sets a distance is assumed to be a dual measure to similarity expressed as Similarity $=1-$ distance.

Definition A distance on a set $X$ is a positive function $d$ (also called a metric) from pairs of elements of $X$ to the set $R^{+}$of non-negative real numbers with the following properties for all $x_{1}, x_{2}, x_{3} \in X$ :

1. $d\left(x_{1}, x_{1}\right)=0$ (reflexivity);

2. $d\left(x_{1}, x_{2}\right)=0$ if and only if $x_{1}=x_{2}$ (identity);

3. $d\left(x_{1}, x_{2}\right)=d\left(x_{2}, x_{1}\right)$ (symmetry);

4. $d\left(x_{1}, x_{3}\right) \leq d\left(x_{1}, x_{2}\right)+d\left(x_{2}, x_{3}\right)$ (triangle inequality).

The pair $(X, d)$ is called a metric space.

We will examine now the effects of using a distance as a dual notion to similarity for A-IFSs.

\subsection{Some intrinsic difficulties}

In Szmidt and Kacprzyk [23] we have shown that the (1-Hamming distance) between the A-IFSs should not be used as a similarity measure between them.

The normalized Hamming distance between the AIFSs $A, B$ in $X=\left\{x_{1}, \ldots, x_{n}\right\}$ (cf. Szmidt and Kacprzyk [15], [19], Szmidt and Baldwin [12, 13]):

$$
\begin{aligned}
l_{I F S}(A, B) & =\frac{1}{2 n} \sum_{i=1}^{n}\left(\left|\mu_{A}\left(x_{i}\right)-\mu_{B}\left(x_{i}\right)\right|+\right. \\
& +\left|v_{A}\left(x_{i}\right)-v_{B}\left(x_{i}\right)\right|+ \\
& \left.+\left|\pi_{A}\left(x_{i}\right)-\pi_{B}\left(x_{i}\right)\right|\right) .
\end{aligned}
$$

For (7) we have: $0 \leq l_{I F S}(A, B) \leq 1$. Clearly the normalized Hamming distance (7) satisfies the conditions of the metric.

The corresponding similarity measure is therefore:

$$
\begin{gathered}
\operatorname{Sim}_{H}=1-l_{I F S}(A, B)= \\
=\quad 1-\frac{1}{2 n} \sum_{i=1}^{n}\left(\left|\mu_{A}\left(x_{i}\right)-\mu_{B}\left(x_{i}\right)\right|+\right. \\
\left.+\quad\left|v_{A}\left(x_{i}\right)-v_{B}\left(x_{i}\right)\right|+\left|\pi_{A}\left(x_{i}\right)-\pi_{B}\left(x_{i}\right)\right|\right) .
\end{gathered}
$$

Figures 2 and 3 show that for a fixed membership value we have elements at the same distance from element $(1,0,0)$. The situation repeats while we examine distances to any element $x:(\mu, v, \pi)$ making use of the normalized Hamming distance. We receive the same type of shapes (Figures 2 and 3) pointing out the elements equally distant from a fixed element.

Now we will demonstrate the result of using the ( 1 normalized Euclidean distance) between the A-IFSs as a similarity measure. 


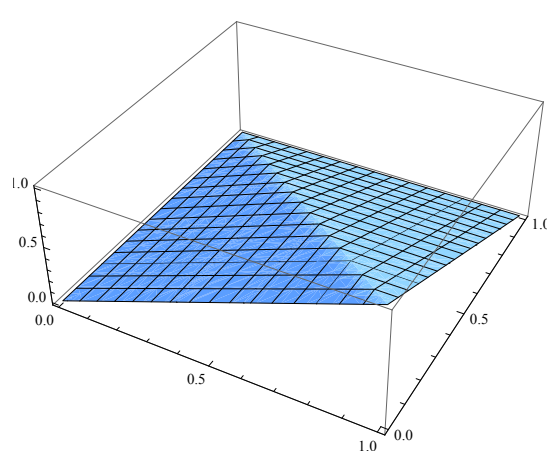

Figure 2: Values of similarity (8) for any element from an A-IFS and element $(1,0,0)$

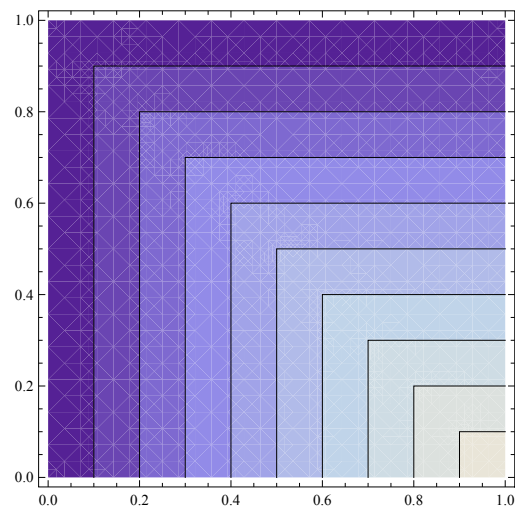

Figure 3: Contourplot of (8) for any element from an A-IFS and element $(1,0,0)$

For the two A-IFS $A$ and $B$ in $X=\left\{x_{1}, x_{2}, \ldots, x_{n}\right\}$, their normalized Euclidean distance is equal to (cf. Szmidt and Kacprzyk [15], [19]):

$$
\begin{aligned}
e_{I F S}(A, B) & =\left(\frac{1}{2 n} \sum_{i=1}^{n}\left(\mu_{A}\left(x_{i}\right)-\mu_{B}\left(x_{i}\right)\right)^{2}+\right. \\
& +\left(v_{A}\left(x_{i}\right)-v_{B}\left(x_{i}\right)\right)^{2}+ \\
& \left.+\left(\pi_{A}\left(x_{i}\right)-\pi_{B}\left(x_{i}\right)\right)^{2}\right)^{\frac{1}{2}}
\end{aligned}
$$

and its counterpart similarity measure would be:

$$
\begin{aligned}
\operatorname{Sim}_{e}(A, B) & =1-e_{I F S}(A, B)= \\
& =1-\left(\left(\frac{1}{2 n} \sum_{i=1}^{n}\left(\mu_{A}\left(x_{i}\right)-\mu_{B}\left(x_{i}\right)\right)^{2}+\right.\right. \\
& +\left(v_{A}\left(x_{i}\right)-v_{B}\left(x_{i}\right)\right)^{2}+ \\
& \left.+\left(\pi_{A}\left(x_{i}\right)-\pi_{B}\left(x_{i}\right)\right)^{2}\right)^{\frac{1}{2}}
\end{aligned}
$$

The results obtained from (10) are illustrated in Figures 4 and 5. Expressing similarity via distances means looking for geometrical shapes, and while using (10) we look in fact for elements at a "radius" distance from a chosen element (object). It is obviously a correct approach (looking for some shapes in a coordinate space) but we should make too far a conclusion about similarity as a dual measure of a distance as it is shown in the following example.

Example For simplicity let us consider "degenerated", "one point type" A-IFS sets which full description is $A=$

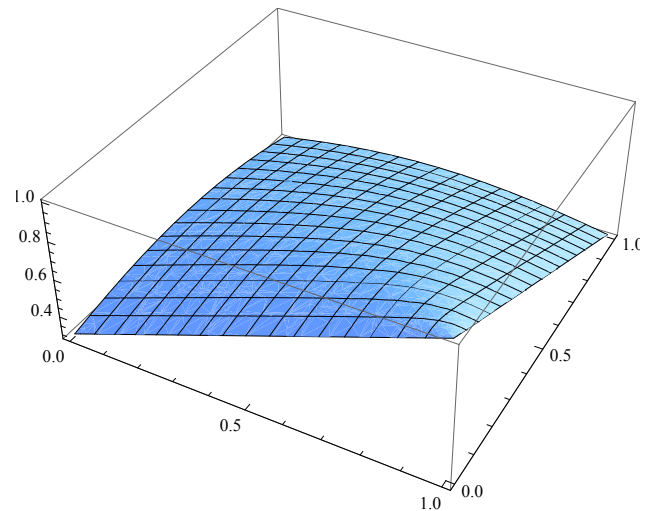

Figure 4: Shape of $\operatorname{Sim}_{e}(A, B)(10)$ for any element from an A-IFS and $(1,0,0)$

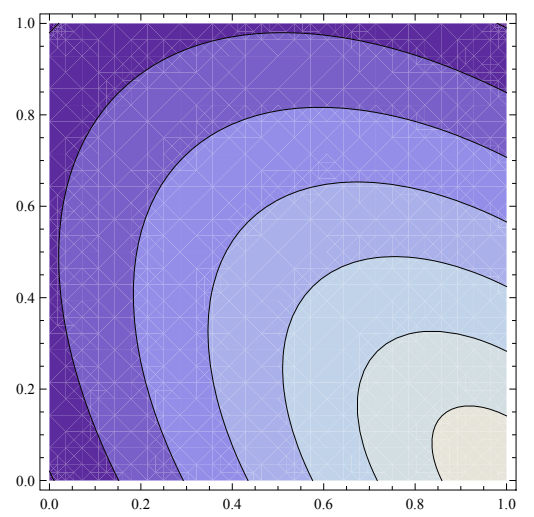

Figure 5: Contourplot of (10) for any element from an A-IFS and $(1,0,0)$

$<x, \mu_{A}, v_{A}, \pi>/ 1$ exemplified by: $M, N, L$ in $X=\{1\}$.

$M=(1,0,0) / 1, \quad N=(0,1,0) / 1, \quad H=(0,0,1) / 1$

From (10) we obtain:

$\operatorname{Sim}_{e}(M, N)=0$, and

$\operatorname{Sim}_{e}(M, H)=0$

though $N$ and $H$ are obviously different. But the "the radius length" from $M$ to $N$ is equal to the "radius length" from $M$ to $H$. It is easy to accept for a crisp case that the elements on a circle are in the same distance from the middle of the circle which does not mean that all the elements belonging to the circle are "the same". Here we have the same situation.

We should also be cautious when considering similarity of the elements with the symmetry of terms in their description, e.g.:

$$
\begin{gathered}
M=(1,0,0) / 1, \quad K=(0.5,0.3,0.2) / 1, \\
L=(0.5,0.2,0.3) / 1
\end{gathered}
$$

for which the exchange of "the places" between nonmembership value and hesitation margin in $K$ and $L$ results in $\operatorname{Sim}_{e}(M, K)=\operatorname{Sim}_{e}(M, L)$ although for sure $K$ and $L$ are different but "the radiuses" $M K$ and $M L$ are the same.

In our previous works (Szmidt and Kacprzyk [15], [19], [25]) we have shown that from a practical point of 
view it is necessary to take into account all three terms describing an A-IFS while calculating distances. But it is interesting to verify the results of "two term distances" between the A-IFSs and their effects as the dual measures to similarity. We will examine the "two term" normalized Hamming and Euclidean distances.

The normalized Hamming distance between the AIFSs $A, B$ in $X=\left\{x_{1}, \ldots, x_{n}\right\}$ while we use two terms only:

$$
\begin{aligned}
l_{H 2 D}(A, B) & =\frac{1}{2 n} \sum_{i=1}^{n}\left(\left|\mu_{A}\left(x_{i}\right)-\mu_{B}\left(x_{i}\right)\right|+\right. \\
& \left.+\left|v_{A}\left(x_{i}\right)-v_{B}\left(x_{i}\right)\right|\right) .
\end{aligned}
$$

For (11) we have: $0 \leq l_{H 2 D}(A, B) \leq 1$. The normalized Hamming distance (11) satisfies the conditions of the metric.

The corresponding similarity measure is:

$$
\begin{gathered}
\operatorname{Sim}_{H 2 D}=1-l_{H 2 D}(A, B)= \\
=1-\frac{1}{2 n} \sum_{i=1}^{n}\left(\left|\mu_{A}\left(x_{i}\right)-\mu_{B}\left(x_{i}\right)\right|+\right. \\
\left.+\left|v_{A}\left(x_{i}\right)-v_{B}\left(x_{i}\right)\right|\right) .
\end{gathered}
$$

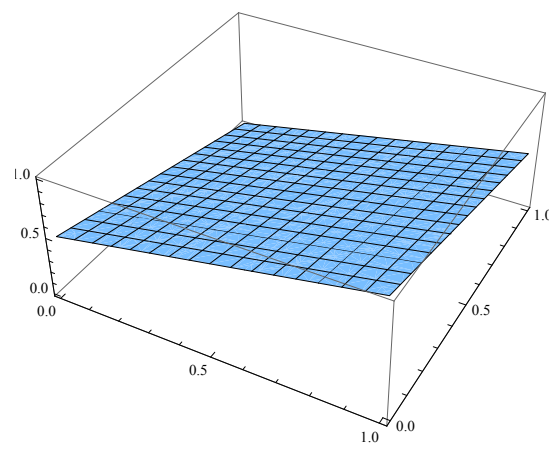

Figure 6: Values obtained from (12) for any element from an A-IFS and $(1,0,0)$

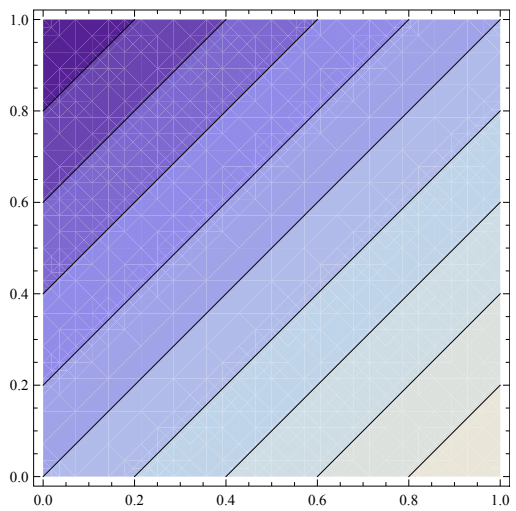

Figure 7: ContourPlot of (12) for any element from and A-IFS and $(1,0,0)$

For the A-IFSs $A$ and $B$ in $X=\left\{x_{1}, x_{2}, \ldots, x_{n}\right\}$, their normalized Euclidean distance while using two terms describing the A-IFSs is:

$$
\begin{aligned}
e_{2 D}(A, B) & =\left(\frac{1}{2 n} \sum_{i=1}^{n}\left(\mu_{A}\left(x_{i}\right)-\mu_{B}\left(x_{i}\right)\right)^{2}+\right. \\
& \left.+\left(v_{A}\left(x_{i}\right)-v_{B}\left(x_{i}\right)\right)^{2}\right)^{\frac{1}{2}}
\end{aligned}
$$

and its corresponding similarity measure would be:

$$
\begin{aligned}
\operatorname{Sim}_{e 2 D}(A, B) & =1-e_{2 D}(A, B)= \\
& =1-\left(\frac{1}{2 n} \sum_{i=1}^{n}\left(\mu_{A}\left(x_{i}\right)-\mu_{B}\left(x_{i}\right)\right)^{2}+\right. \\
& \left.+\left(v_{A}\left(x_{i}\right)-v_{B}\left(x_{i}\right)\right)^{2}\right)^{\frac{1}{2}}
\end{aligned}
$$

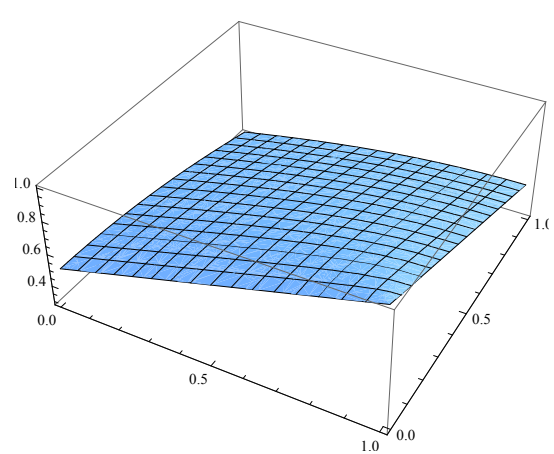

Figure 8: Values obtained from (14) for any element from A-IFS and $(1,0,0)$

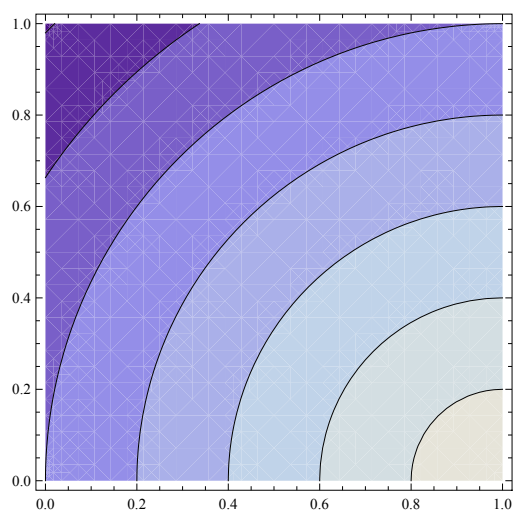

Figure 9: ContourPlot of (14) for any element from AIFS and $(1,0,0)$

Figures 6-9 show that by making use of two term distances as dual concepts of similarity measures the situation does not change in the sense of the information obtained (certainly we do not suggest here that in general both ways of A-IFSs representations are equal having in mind other drawbacks of two term representation as compared to three term representation of A-IFSs - cf. Szmidt and Kacprzyk [25]).

Another similarity measure that is often used in practice is the cosine similarity measure which is based on Bhattacharya's distance [5], [10] and is expressed as the inner product of two vectors divided by the product of their lengths. In other words it is the cosine of the angle between two vectors. The cosine similarity is often used 
in information retrieval [10]. Taking as a point of departure the three term A-IFS representation, the cosine similarity measure is given by (15).

$$
\begin{array}{ll} 
& \operatorname{Sim}_{\text {mult }}(A, B)=\frac{1}{n} \sum_{i=1}^{n}\left(\left(\mu_{A}\left(x_{i}\right) \mu_{B}\left(x_{i}\right)+\right.\right. \\
+\quad & \left.v_{A}\left(x_{i}\right) v_{B}\left(x_{i}\right)+\pi_{A}\left(x_{i}\right) \pi_{B}\left(x_{i}\right)\right) / \\
/ \quad & \left(\mu_{A}\left(x_{i}\right)^{2}+v_{A}\left(x_{i}\right)^{2}+\pi_{A}\left(x_{i}\right)^{2}\right)^{\frac{1}{2}} \\
& \left.\left.\left(\mu_{B}\left(x_{i}\right)^{2}+v_{B}\left(x_{i}\right)^{2}+\pi_{B}\left(x_{i}\right)^{2}\right)\right)^{\frac{1}{2}}\right)
\end{array}
$$

In Figures 10 and 11 there are values obtained from (15) which illustrate similarity of element $(1,0,0)$ and any other element.

It is worth mentioning again that in (15) we might change the places of $v$ and $\pi$, and the result of (15) will be the same although we consider quite different situations. This is clearly some undesired effect.

Example Let us consider again "degenerated" A-IFS sets the full description of which is $A=\left\{\left\langle x, \mu_{A}, v_{A}, \pi\right\rangle\right.$ $/ 1\}$ exemplified by: $M, R, S$ in $X=\{1\}$. Where $M=(1,0,0) / 1$, and

$$
R=(0.5,0.3,0.2) / 1, \quad S=(0.5,0.2,0.3) / 1
$$

From (15) we obtain:

$\operatorname{Sim}_{\text {mult }}(M, R)=\operatorname{Sim}_{\text {mult }}(M, S)=0.81$ whereas $R$ and $S$ are obviously different, so we assume that their similarity to the same $M$ should be different, too.

Measure (15) is not able to differentiate, e.g., between: $(0,0,1)$, and $(0,1,0)$ while examining their similarity to $(1,0,0)$ (cf. Figures 10 and 11). Certainly, we may point out many such cases in respect to (15).

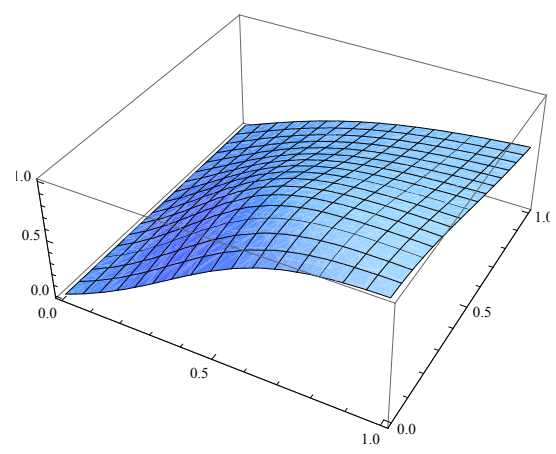

Figure 10: Values obtained from measure (15) for any element from an A-IFS and $(1,0,0)$

It is necessary to emphasize again that the above measures give correct answers in the sense of the formula used (geometrical shapes are recognized in respect to a chosen element) but in many situations we would expect that from the similarity measures we would be able at least to notice the existence of the complement element which seems to be the least similar to the considered element.

The problem of symmetry between the membership, non-membership and hesitation margin (cf. Szmidt and Kreinovich [26]) in the above formulas can be partly



Figure 11: Contourplot of measure (15) for any element from an A-IFS and $(1,0,0)$

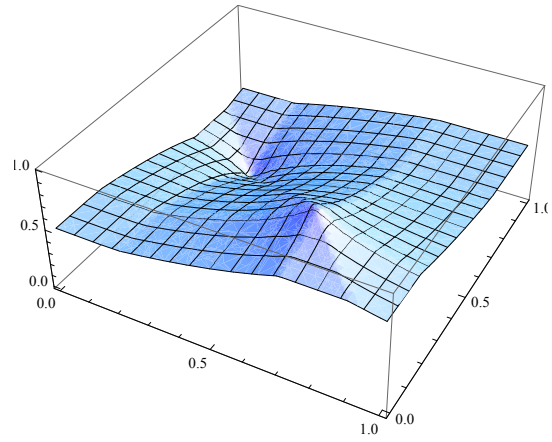

Figure 12: Values obtained from measure (16) for any element from an A-IFS and $(0.7,0.2,0.1)$

removed by introducing into these definitions of the measures of similarity not only a relation to an element we are interested in but also that to its complement. The following formulas were proposed (Szmidt and Kacprzyk [19], [21], [24]).

$$
\begin{aligned}
& \operatorname{Sim}\left(l_{I F S}(X, F), l_{I F S}\left(X, F^{C}\right)\right)= \\
& =1-f\left(l_{I F S}(X, F), l_{I F S}\left(X, F^{C}\right)\right)= \\
& =1-\frac{l_{I F S}(X, F)}{l_{I F S}(X, F)+l_{I F S}\left(X, F^{C}\right)} \text {, } \\
& \operatorname{Sim}\left(l_{I F S}(X, F), l_{I F S}\left(X, F^{C}\right)\right)= \\
& =\frac{1-f\left(l_{I F S}(X, F), l_{I F S}\left(X, F^{C}\right)\right)}{1+f\left(l_{I F S}(X, F), l_{I F S}\left(X, F^{C}\right)\right)}, \\
& \operatorname{Sim}\left(l_{I F S}(X, F), l_{I F S}\left(X, F^{C}\right)\right)= \\
& =\frac{1-f\left(l_{I F S}(X, F), l_{I F S}\left(X, F^{C}\right)\right)^{2}}{1+f\left(l_{I F S}(X, F), l_{I F S}\left(X, F^{C}\right)\right)^{2}} \\
& \operatorname{Sim}\left(l_{I F S}(X, F), l_{I F S}\left(X, F^{C}\right)\right)= \\
& =\frac{e^{-f\left(l_{I F S}(X, F), l_{I F S}\left(X, F^{C}\right)\right)}-e^{-1}}{1-e^{-1}},
\end{aligned}
$$

where

$$
\begin{gathered}
f\left(l_{I F S}(X, F), l_{I F S}\left(X, F^{C}\right)\right)= \\
=\frac{l_{I F S}(X, F)}{l_{I F S}(X, F)+l_{I F S}\left(X, F^{C}\right)} .
\end{gathered}
$$




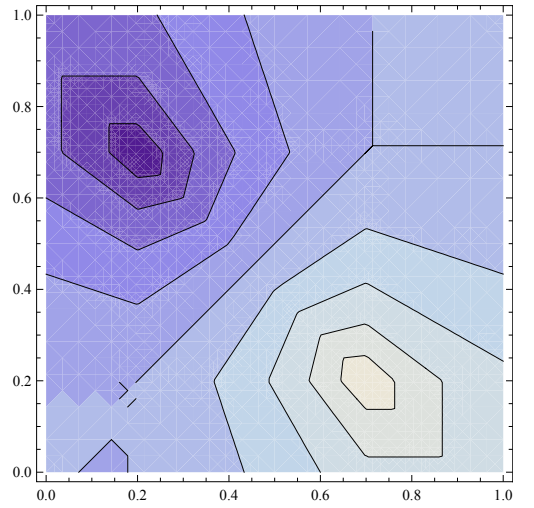

Figure 13: Contourplot of measure (16) for any element from an A-IFS and $(0.7,0.2,0.1)$

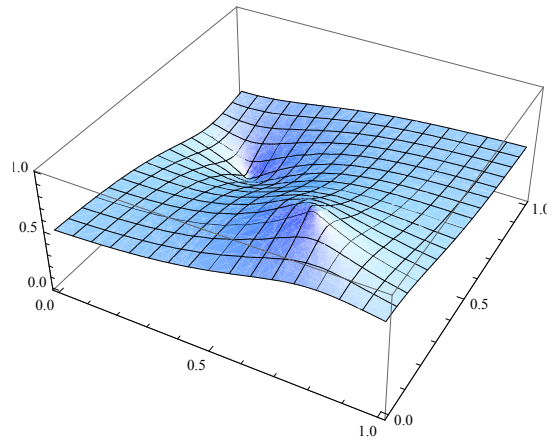

Figure 14: Values obtained from (22) for any element from an A-IFS and $(0.7,0.2,0.1)$

Function $f\left(l_{I F S}(X, F), l_{I F S}\left(X, F^{C}\right)\right)$ (20) making use of the Hamming distance in (16) - (19) can be replaced by its corresponding function making use of the Euclidean distance, i.e.:

$$
\begin{gathered}
f\left(e_{I F S}(X, F), e_{I F S}\left(X, F^{C}\right)\right)= \\
=\frac{e_{I F S}(X, F)}{e_{I F S}(X, F)+e_{I F S}\left(X, F^{C}\right)}
\end{gathered}
$$

where $e_{I F S}(X, F)$ is given by (9). For example, the counterpart of (16) with (21) instead of (20) is:

$$
\begin{aligned}
& \operatorname{Sim}\left(e_{I F S}(X, F), e_{I F S}\left(X, F^{C}\right)\right)= \\
= & 1-f\left(e_{I F S}(X, F), e_{I F S}\left(X, F^{C}\right)\right)= \\
= & 1-\frac{e_{I F S}(X, F)}{e_{I F S}(X, F)+e_{I F S}\left(X, F^{C}\right)} .
\end{aligned}
$$

In Figures $14-15$ we have an illustration of the results from (22). The formulas with (21) give analogical results to $(16)-(19$ in the sense of pointing out some geometrical "shapes" but still the problem of symmetry concerning terms (describing an A-IFS) in the formulas was not completely solved as quite different elements from the point of view of, for instance, decision making are "the same" in the sense of the values obtained from the proposed measures in respect to a chosen element. A simple "weighting" of the terms describing the elements does not solve the problem either. In Figures 16 and 17 we have results from (22) with "weighted" membership values (in Figure 16 the membership value is

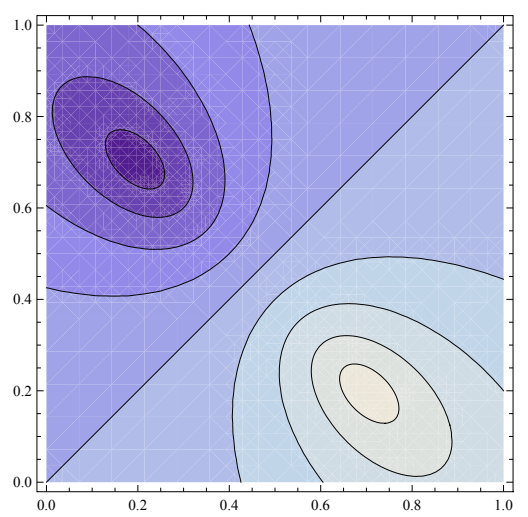

Figure 15: Contourplot of (22) for any element from an A-IFS and $(0.7,0.2,0.1)$

two times more important, and in Figure 17 ten times more important than the non-membership and hesitation margin). The geometrical shapes pointed out by the weighted similarity measures change (cf. Figures 15, 16 and 17).

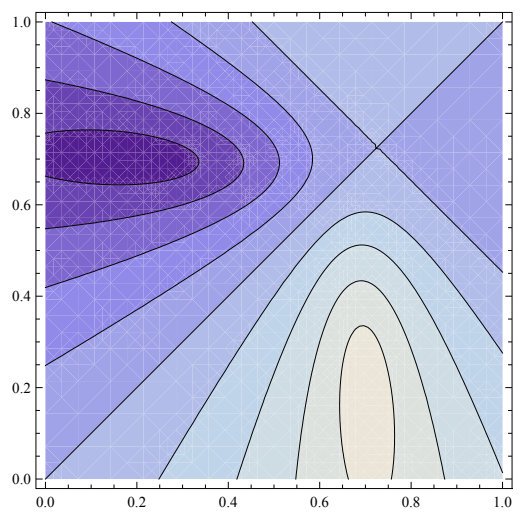

Figure 16: Contourplot of (22) (with two times more important membership values) for any element from an A-IFS and $(0.7,0.2,0.1)$

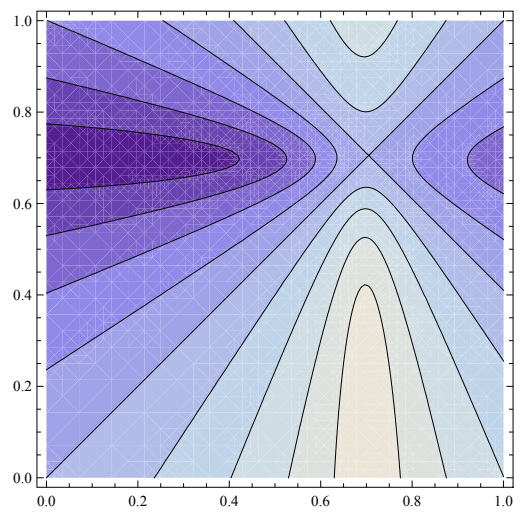

Figure 17: Contourplot of (22) (with ten times more important membership values) for any element from an AIFS and $(0.7,0.2,0.1)$

The question arises what should be done if we wish to use similarity measure (22) and to differentiate between elements $(0.3,0,0.7)$ and $(0.5,0.4,0.1)$ which 
are obviously different from the point of view of decision making but both are similar to element $(0.7,0.2$, 0.1 ) to the same extent equal to 0.6 (cf. Figures 14 and 15). First, we should not conclude about similarity of $(0.3,0,0.7)$ and $(0.5,0.4,0.1)$ before calculating their similarity from $(22)$ - we obtain the value 0.51 (different from 0.6).

Now we will examine another definition of similarity using the Hausdorff distance (cf. Grünbaum [9]). The Hausdorff distance is the maximum distance of a set to the nearest point in the other set. A more formal description is given by the following

Definition Given two finite sets $A=\left\{a_{1}, \ldots, a_{p}\right\}$ and $B=\left\{b_{1}, \ldots, b_{q}\right\}$, the Hausdorff distance $H(A, B)$ is defined as:

$$
H(A, B)=\max \{h(A, B), h(B, A)\}
$$

where

$$
h(A, B)=\max _{a \in A} \min _{b \in B} d(a, b)
$$

where:

- $a$ and $b$ are elements of sets $A$ and $B$, respectively,

$-d(a, b)$ is any metric between these elements,

- the two distances $h(A, B)$ and $h(B, A)(24)$ are called the directed Hausdorff distances.

In Szmidt and Kacprzyk [25] we have shown that in a case of the Hamming distance we should use the following formula (with all three terms describing an A-IFS):

$$
\begin{aligned}
& H_{3}(A, B)=\frac{1}{n} \sum_{i=1}^{n} \max \left\{\left|\mu_{A}\left(x_{i}\right)-\mu_{B}\left(x_{i}\right)\right|,\right. \\
& \left.\left|v_{A}\left(x_{i}\right)-v_{B}\left(x_{i}\right)\right|,\left|\pi_{A}\left(x_{i}\right)-\pi_{B}\left(x_{i}\right)\right|\right\}
\end{aligned}
$$

If we apply distance (25) in the formulas (16) - (19) instead of (20), we obtain new similarity measures. For example, the counterpart of (16) with (25) instead of (20) is:

$$
\begin{aligned}
& \operatorname{Sim}\left(H_{3}(X, F), H_{3}\left(X, F^{C}\right)\right)= \\
= & 1-f\left(H_{3}(X, F), H_{3}\left(X, F^{C}\right)\right)= \\
= & 1-\frac{H_{3}(X, F)}{H_{3}(X, F)+H_{3}\left(X, F^{C}\right)} .
\end{aligned}
$$

In Figures 18 and 19 we have results from (26)



Figure 18: Values of similarity (26) for any element from an A-IFS and element $(0.7,0.2,0.1)$

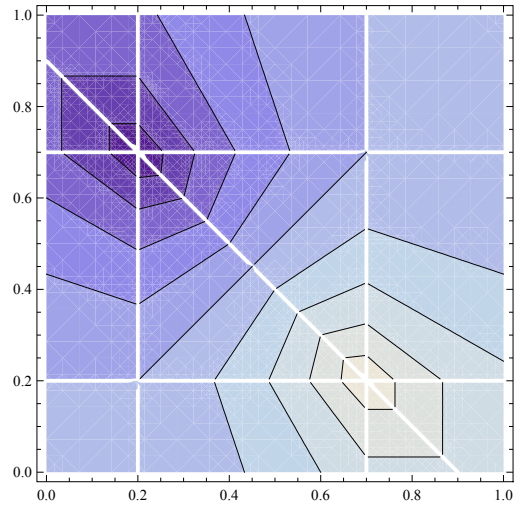

Figure 19: Contourplot of measure (26) for any element from an A-IFS and element $(0.7,0.2,0.1)$

The accounting for the complement elements in the similarity measures seems important in many tasks (for example in image recognition the most "dissimilar" image is a negative image which can be understood as an image consisting of the complement elements).

\section{Conclusions}

We have considered several possible geometric similarity measures between the A-IFSs. Our remarks are general but should be taken into account to obtain reliable results. First, it is necessary to have in mind the symmetry in the description of the A-IFS elements, and the fact that from the knowledge of the geometric similarity of a fixed element to two different elements we should not conclude about the similarity of the two elements (their similarity should be examined). We have also pointed out that while defining similarity, viewing it just as a a dual concept of a distance is not enough and the use of the complement elements help attain more intuitively appealing and reliable results.

\section{Acknowledgment}

Partially supported by the National Science Centre.

\section{References}

[1] Atanassov K. (1983) Intuitionistic Fuzzy Sets. VII ITKR Session. Sofia (Deposed in Centr. Sci.Techn. Library of Bulg. Acad. of Sci., 1697/84) (in Bulgarian).

[2] Atanassov K. (1986) Intuitionistic Fuzzy Sets. Fuzzy Sets and Systems, 20, 87-96.

[3] Atanassov K. (1999) Intuitionistic Fuzzy Sets: Theory and Applications. Springer-Verlag.

[4] Atanassov K. (2012) On Intuitionistic Fuzzy Sets Theory. Springer-Verlag.

[5] Bhattacharya A. (1946) On a measure of divergence of two multinomial populations, Sankhya 7, 401-406. 
[6] Bustince H., Mohedano V., Barrenechea E., and Pagola M. (2005) Image thresholding using intuitionistic fuzzy sets. In: Issues in the Representation and Processing of Uncertain and Imprecise Information. Fuzzy Sets, Intuitionistic Fuzzy Sets, Generalized Nets, and Related Topics. (Eds. Atanassov K., Kacprzyk J., Krawczak M., Szmidt E.), EXIT, Warsaw 2005.

[7] Bustince H., Mohedano V., Barrenechea E., and Pagola M. (2006) An algorithm for calculating the threshold of an image representing uncertainty through A-IFSs. IPMU'2006, 2383-2390.

[8] Carroll, J. D., Wish, M. (1974) Multidimensional perceptual models and measurement methods. In E. C. Carterette and M. P. Friedman (Eds.), Handbook of perception. New York: Academic Press.

[9] Grünmaum B. (1967) Convex Polytopes, WileyInterscience, New York.

[10] Salton G., McGill M. J. (1983) Introduction to Modern Information Retrieval, McGraw-Hill Book Company, New York.

[11] Shepard, R. N. Representation of structure in similarity data: Problems and prospects. Psychomelrika, 1974, 39, 373-421.

[12] Szmidt E. and Baldwin J. (2003) New Similarity Measure for Intuitionistic Fuzzy Set Theory and Mass Assignment Theory. Notes on IFSs, 9(3), 60 76.

[13] Szmidt E. and Baldwin J. (2004) Entropy for Intuitionistic Fuzzy Set Theory and Mass Assignment Theory. Notes on IFSs, 10(3), 15-28.

[14] Szmidt E. and Kacprzyk J. (1997) On measuring distances between intuitionistic fuzzy sets, Notes on IFS, 3(4), 1-13.

[15] Szmidt E. and Kacprzyk J. (2000) Distances between intuitionistic fuzzy sets, Fuzzy Sets and Systems, 114(3), 505-518.

[16] Szmidt E., Kacprzyk J. (2001) Entropy for intuitionistic fuzzy sets. Fuzzy Sets and Systems, 118 (3), 467-477.

[17] Szmidt E. and Kacprzyk J. (2004) Similarity of intuitionistic fuzzy sets and the Jaccard coefficient. IPMU 2004, 1405-1412.

[18] Szmidt E., Kacprzyk J. (2004) A Concept of Similatity for Intuitionistic Fuzzy Sets and its use in Group Decision Making. 2004 IEEE Conf. on Fuzzy Systems, Budapest, 1129-1134.

[19] Szmidt E. and Kacprzyk J. (2006) Distances Between Intuitionistic Fuzzy Sets: Straightforward Approaches may not work. 3rd Int. IEEE Conf. Intelligent Systems IS06, London, 716-721.

[20] Szmidt E. and Kacprzyk J. (2006) An Application of Intuitionistic Fuzzy Set Similarity Measures to a Multi-criteria Decision Making Problem. ICAISC 2006, LNAI 4029, Springer-Verlag, 314-323.

[21] Szmidt E. and Kacprzyk J. (2007a). A New Similarity Measure for Intuitionistic Fuzzy Sets: Straightforward Approaches may not work. 2007 IEEE Conference on Fuzzy Systems, 481-486.

[22] Szmidt E. and Kacprzyk J. (2007) Some problems with entropy measures for the Atanassov intuitionistic fuzzy sets. Applications of Fuzzy Sets Theory. LNAI 4578, 291-297. Springer-Verlag.

[23] Szmidt E. and Kacprzyk J. (2009) Amount of information and its reliability in the ranking of Atanassov's intuitionistic fuzzy alternatives. In: Recent Advances in decision Making, SCI 222. E. Rakus-Andersson, R. Yager, N. Ichalkaranje, L.C. Jain (Eds.), Springer-Verlag, 7-19.

[24] Szmidt E., Kacprzyk J. (2009) Analysis of Similarity Measures for Atanassov's Intuitionistic Fuzzy Sets. In: Proceedings IFSA/EUSFLAT 2009, 1416-1421.

[25] Szmidt E., Kacprzyk J. (2011) Intuitionistic fuzzy sets - Two and three term representations in the context of a Hausdorff distance. Acta Universitatis Matthiae Belii, Series Mathematics, available at HTTP://ACTAMTH.SAVBB.SK, Vol.19, No. 19, 2011, 53-62.

[26] Szmidt E., Kreinovich V. (2009) Symmetry between true, false, and uncertain: An explanation. Notes on Intuitionistic Fuzzy Sets 15, No. 4, 1-8.

[27] Szmidt E. and Kukier M. (2006) Classification of Imbalanced and Overlapping Classes using Intuitionistic Fuzzy Sets. IEEE IS'06, London, 722727.

[28] Szmidt E. and Kukier M. (2008) A New Approach to Classification of Imbalanced Classes via Atanassov's Intuitionistic Fuzzy Sets. In: HsiaoFan Wang (Ed.): Intelligent Data Analysis : Developing New Methodologies Through Pattern Discovery and Recovery. Idea Group, 85-101.

[29] Szmidt E., Kukier M. (2008) Atanassov's intuitionistic fuzzy sets in classification of imbalanced and overlapping classes. In: Panagiotis Chountas, Ilias Petrounias, Janusz Kacprzyk (Eds.): Intelligent Techniques and Tools for Novel System Architectures. Springer, Berlin Heidelberg 2008, 455-471. Seria: Studies in Computational Intelligence.

[30] Tversky A. (1977) Features of similarity. Psychological Rev., 84, 4, 327-352.

[31] Veltkamp R.C., Hagedoorn M. (2000) Shape similarities, properties, and constructions. In Advances in Visual Information Systems, Proc. 4th International Conference, VISUAL 2000, LNCS 1929, Springer, 467-476.

[32] Veltkamp R.C. (2001) Shape Matching: Similarity Measures and Algorithms. UU-CS-2001-3, 1-17.

[33] Ye J. (2011) Cosine similarity measures for intuitionistic fuzzy sets and their applications. Mathematical and Computer Modelling 53, 91-97.

[34] Zadeh L.A. (1965) Fuzzy sets. Inf. and Control, 8, 338-353. 